\title{
Development of Postoperative Pneumonia After Endovascular Aortic Aneurysm Repair is Associated with an Increased Length of Intensive Care Unit Stay
}

\author{
Cam Dung Le ${ }^{1}$, Erik Lehman ${ }^{1}$, Faisal Aziz ${ }^{2}$ \\ 1. Surgery, Penn State College of Medicine, Penn State Milton S. Hershey Medical Center, Hershey, USA 2. \\ Cardiac / Thoracic / Vascular Surgery, Penn State College of Medicine, Penn State Milton S. Hershey \\ Medical Center, Hershey, USA
}

$\square$ Corresponding author: Cam Dung Le, camdungle@gmail.com

Disclosures can be found in Additional Information at the end of the article

\section{Abstract \\ Objective}

Endovascular aortic aneurysm repair (EVAR) has increasingly replaced open aortic surgery for treatment of abdominal aortic aneurysms (AAA). One of the key advantages of EVAR is the reduced length of intensive care unit (ICU) stay and hospital stay. This study aimed to identify the risk factors associated with increased ICU length of stay (LOS) after EVAR.

\section{Methods}

The American College of Surgeons (ACS-NSQIP) database for the year 2013 was used. All patients who underwent EVAR were divided into two groups: ICU LOS $<1$ day vs. $\geqslant 1$ day. Preoperative, intraoperative, and postoperative factors were compared between these two groups utilizing bivariate logistic regression analysis. Multivariable logistic regression analysis was then used to identify factors that were independently associated with ICU LOS $\geqslant 1$ day after EVAR.

\section{Results}

A total of 2,468 patients (18.7\% females, $81.3 \%$ males) were identified. Group 1 (ICU LOS <1 day) $=1,535$ patients and Group 2 (ICU LOS $\geqslant 1$ day) $=933$ patients. Multivariable analysis identified the following factors to be associated with ICU LOS $\geqslant 1$ day: ruptured AAA (OR 3.88, CI 1.97-7.65), the American Society of Anesthesiology (ASA) score of 4-5 (OR 2.82, CI 1.50-5.31), operative time $\geqslant 180$ minutes (OR 2.10, CI 1.51-2.93), bilateral groin cut down (OR 1.37, CI 1.10-

Received 01/28/2019 Review began 02/26/2019 Review ended 04/11/2019 Published 04/21/2019

\section{(c) Copyright 2019}

Le et al. This is an open access article distributed under the terms of the Creative Commons Attribution License CC-BY 3.0., which permits unrestricted use, distribution, and reproduction in any medium, provided the original author and source are credited. 1.71), juxta-renal AAA (OR 1.65, CI 1.16-2.35), renal artery stent (OR 2.13, CI 1.42-3.21), aortic stent (OR 2.39, CI 1.60-3.55), emergency surgery (OR 2.56, CI 1.94-3.38), need for blood transfusion (OR 3.11, CI 2.08-4.65) and postoperative pneumonia (OR 7.04, CI 1.95-25.45).

\section{Conclusion}

Variables identified above can be used to predict the cohort of EVAR patients which will likely require ICU for $\geqslant 1$ day. Development of postoperative pneumonia is associated with a 7.04 times increase in ICU LOS $\geqslant 1$ day.

Categories: Cardiac/Thoracic/Vascular Surgery, General Surgery

How to cite this article

Le C, Lehman E, Aziz F (April 21, 2019) Development of Postoperative Pneumonia After Endovascular Aortic Aneurysm Repair is Associated with an Increased Length of Intensive Care Unit Stay. Cureus 11(4): e4514. DOI 10.7759/cureus. 4514 
Keywords: endovascular aneurysm repair (evar), pneumonia, abdominal aortic aneurysm (aaa), icu length of stay

\section{Introduction}

The past two decades have seen a revolution in the surgical treatment of abdominal aortic aneurysms (AAA). Since EVAR was first described by Parodi et al., subsequent randomized controlled trials have supported its safety and efficacy [1-3]. EVAR has now become the most common modality for the treatment of abdominal aortic aneurysms. One of the main advantages of EVAR over traditional open AAA repair is the reduced intensive care unit (ICU) and hospital length of stay (LOS). There is recent evidence to suggest that EVAR can be performed safely even in the outpatient settings [4]. In the era of increasing focus on reducing the postoperative complications, the length of stay in the intensive care unit and hospital, and healthcare costs, hospitals are developing strategies to improve these metrics.

Most patients do not need ICU care after elective EVAR. However, patients with numerous physiologic risk factors and those who undergo emergent EVAR are in need of ICU care postoperatively. Increased length of ICU stay after EVAR has been shown to be associated with significant complications, including death [5]. In this study, we aimed to determine the associating factors and develop a risk model for predicting the increased ICU LOS after EVAR. For this purpose, we chose to use the American College of Surgeons National Surgical Quality Improvement Program (ACS-NSQIP), since this is the largest surgical database in the US with a record of ICU LOS for a variety of general and vascular surgery operations.

\section{Materials And Methods}

\section{Dataset}

For the purpose of this study, we retrospectively analyzed the database provided by the American College of Surgeons National Surgical Quality Improvement Program (ACSNSQIP). This database provides the users with Participant Use Files (ACS-NSQIP PUFs) [6]. These files contain patient de-identified data from multiple hospitals that participate in the ACS-NSQIP. ACS ensures that the information in the database remains compliant with the requirements of the Health Insurance Portability and Accountability Act (HIPAA). The database contains data from over 250 hospitals across the United States. It contains over 150 patient variables, including preoperative, intraoperative and postoperative factors. In order to ensure the integrity of the data, ACS requires each site to hire a reviewer who is specifically trained to collect and enter the data into the database. Data are collected in a systematic sampling process using an eight-day cycle schedule to avoid bias in selecting cases. To maintain diversity and high-quality data, high volume procedures are limited to a certain maximum number of cases per cycle, and cases with incomplete 30-day follow up are excluded from the database. The database is reliable with the inter-rater reliability (IRR) audit of the overall disagreement rate of approximately $2 \%$ among the participating sites [7]. Methods used for this analysis have been described in the previously published literature [7-11].

\section{Outcomes}

The main ACS NSQIP PUF was merged with the EVAR-Procedure Targeted PUF to identify patients who underwent EVAR in the calendar year 2015. Patients were divided into two groups based on LOS in the ICU: Group 1 (ICU LOS < 1 day) and Group 2 (ICU LOS $\geqslant 1$ day). Several preoperative, intra-operative and post-operative variables were compared between these two groups.

\section{Statistical analysis}


All variables were summarized prior to any analysis using frequencies and percentages or means, medians, and standard deviations to examine data quality. The distribution of continuous variables was checked using histograms, box plots, and normal probability plots. Logistic regression was applied using a bivariate approach to search for associations between ICU LOS $\geqslant 1$ and potential predictor variables. Because there were so many significant variables, only variables with a significant association with ICU LOS $\geqslant 1$ having a $p$-value $<0.01$ were chosen as a subset for consideration for a multivariable predictor model. Before model selection was initiated, a check for multicollinearity between the potential predictor variables was performed using a variance inflation factor (VIF) statistics. The remaining predictors were then pared down to a final model using several methods of selection: best subsets, stepwise, backward, and forward. Inclusion and exclusion criteria for the selection methods that used them were set at $p<0.01$ to be more stringent, given the large number of significant predictors. The results of these selection methods were combined to choose the most significant set of predictors of ICU LOS $\geqslant 1$ collectively. The fit of the final model was assessed using Pearson, Deviance, and the Hosmer and Lemeshow goodness-of-fit tests. Model-adjusted odds ratios were used to quantify the size and direction of the significant associations. Predicted probabilities based on patient characteristics were calculated using the prediction equation created based on the model parameter estimates. All analyses were carried out using SAS version 9.4 (SAS Institute, Cary, NC).

\section{Results}

\section{Demographics}

A total of 2,512 patients underwent EVAR in 2015. The mean age was $73.8 \pm 9.0$ years. ICU LOS could be determined in only 2,468 (18.7\% females, $81.3 \%$ males) of these patients and were included in the analysis. Of these 2,468 patients, 1535 had ICU LOS $<1$ day (Group 1) and 933 had ICU LOS $\geqslant 1$ day (Group 2). The mean ICU LOS was $1.0( \pm 2.8)$ days.

\section{Bivariate analysis}

The following factors were found to have significant association with ICU LOS $\geqslant 1$ day: sex (female vs male: odd ratio (OR) 1.26, confidence interval (CI) 1.03-1.55, $p=0.028$, ), race (Hispanic vs non-Hispanic white: OR 3.93, CI 1.95-7.92, $p<0.001$ ), body mass index (BMI; $\geqslant 30$ vs <25: OR 0.77, CI 0.63-0.95, $p=0.004$ ), indication for surgery (ruptured vs non-ruptured: OR 9.86, CI 6.65-14.64, $p<0.001$ ), transferred status (OR 4.57, CI 3.54-5.91, $p<0.001$ ), emergency surgery (OR 4.71, CI 3.87-5.73, $p<0.001$ ), dependent functional health status prior to surgery (OR 2.48, CI 1.56-3.94, $p<0.001$ ), history of chronic obstructive pulmonary disease (COPD; OR 1.58, CI 1.29-1.93, $p<0.001$ ), congestive heart failure (CHF; OR 3.58, CI 2.03-6.31, $p<0.001$ ), hypertension (HTN) requiring medication (OR 1.27, CI 1.04-1.56, $p=0.022$ ), preoperative transfusion (OR 8.41, CI 4.49-15.75, $p<0.001$ ), systemic sepsis (OR 6.43, CI 3.89-10.62, $p<$ 0.001 ), wound classification (clean vs not clean OR 0.24, CI 0.09-0.62, $p=0.003$ ), surgeon's specialty (non-vascular vs vascular: OR 2.00, CI 1.23-3.25, $p=0.005$ ), principal operative procedure (EVAR with aorto-aortic tube prosthetic vs EVAR with modular bifurcated prosthetic with one docking limb: OR 2.19, CI 1.52-3.14, $p<0.001$ ), American Society of Anesthesiologists (ASA) classification (class 4-5 vs class 1-2: OR 4.69, CI 2.73-8.06, $p<0.001$ ), rupture of aneurysm (OR 14.91, CI 1.89-117.67, $p=0.010$ ), aneurysm diameter (cm; >6.0 vs $\leqslant 5.0$ : OR 1.50, CI 1.19$1.88, p<0.001$ ), proximal aneurysm extent (others vs infrarenal: OR 2.73, CI 2.08-3.58, $p<$ 0.001 ), distal extent (internal iliac vs aortic: OR 2.40, CI 1.74-3.30, $p<0.001$ ), access vessels (OR 2.25, CI 1.51-3.35, $p<0.001$ ), access (bilateral groin cutdown vs percutaneous bilateral: OR 1.48, CI 1.25-1.76, $p<0.001$ ), main body device (regular EVARs vs Cook Zenith Fenestrated: OR 0.47, CI 0.29-0.75, $p=0.001$ ), iliac branched device (OR 1.37, CI 1.09-1.72, $p=0.007$ ), aortic stent (OR 1.89, CI 1.38-2.59, $p<0.001$ ), renal stent (OR 3.35, CI 2.45-4.57, $p<0.001$ ), hypogastric revascularization (OR 3.12, CI 2.22-4.39, $p<0.001$ ), lower extremity revascularization (OR 2.00, CI 1.37-2.92, $p<0.001$ ), ischemic colitis (OR 42.11, CI 5.71-310.39, $p<0.001$ ), lower extremity 


\section{Cureus}

ischemia (OR 2.13, CI 1.14-3.97, $p=0.017$ ), operation time (minutes; $\geqslant 180$ vs <90: OR 3.89, CI 3.03-5.00, $p<0.001$ ), discharge destination (home vs expired: OR 8.33, CI 4.42-15.72, $p<$ 0.001), deep incisional surgical site infection (SSI; OR 11.59, CI 1.42-94.32, $p=0.022$ ), pneumonia (OR 17.14, CI 6.11-48.08, $p<0.001$ ), pulmonary embolism (OR 11.59, CI 1.42-94.32, $p=0.022$ ), acute renal failure (OR 38.69, CI 5.23-286.45, $p<0.001$ ), cerebrovascular accident (CVA)/stroke (OR 7.47, CI 1.61-34.63, $p=0.010$ ), cardiac arrest (OR 4.29, CI 2.12-8.65, $p<0.001$ ), myocardial infarction (MI; OR 9.64, CI 4.03-23.04, $p<0.001$ ), bleeding requiring transfusion (OR 9.16, CI 6.80-12.33, $p<0.001$ ), deep vein thrombosis (DVT; OR 9.99, CI 2.23-44.71, $p=0.003$ ), sepsis (OR 4.15, CI 1.30-13.26, $p=0.017)$, return to the operation room (OR 3.12, CI 2.15-4.52, $p<0.001$ ), and unplanned readmission (OR 2.27, CI 1.68-3.07, $p<0.001$; Table 1).

\section{Variable}

Preoperative factors:

Age (years)

$<60$

60-69

$70-79$

$\geq 80$

Sex

Female

461

$266(57.7)$

2007

Male

Race

Hispanic

40 (2.0)

$11(27.5)$

$133(6.6)$

$64(48.1)$

$31(1.5)$

$14(45.2)$

1821

(89.9)
Group 2 ICU LOS

$\geq 1$ day $(\mathrm{N}=933)$

\section{OR $(95 \%$ CI)*}

P. value*
Non-Hispanic White

Non-Hispanic Black

Non-Hispanic Other

BMI $\left(\mathrm{kg} / \mathrm{m}^{\wedge} 2\right)$

$<25$ (normal)
$374(57.1)$

Reference

$0.79(0.55$

1.15)

0.166

$0.69(0.48$, $0.98)$

$0.75(0.52$ 1.08)

Reference

3.93

$(1.95,7.92)$

$1.61(1.13$

2.29)

$<0.001$

$1.81(0.89$

3.70)

Reference 


\section{Cureus}

25-<30 (overweight)

>30 (obese)

Ruptured indication for surgery

Ruptured

Non-ruptured

Transferred

Yes

No

Elective surgery

Yes

No

Diabetes (with oral agents or insulin)

No

Non-insulin dependent

Insulin dependent

Current smoker within one year

Yes

No

2080

(84.3)

$1308(62.9)$

$304(12.3) \quad 184(60.5)$

$84(3.4)$

$43(51.2)$

$41(48.8)$

$332(40.2)$

1641

(66.5)

$1040(63.4)$

$601(36.6)$

Dyspnea

$\begin{array}{ll}\text { Yes } & 378 \\ & \\ \text { No } & 2090\end{array}$

$378(15.3) \quad 222(58.7)$

2090

(84.7)
$1.16(0.98$,

1.38)

0.089

Reference

$0.72(0.58$

$0.88)$

$0.77(0.63$

$0.95)$

9.86

(6.65,

$<0.001$

14.64)

Reference

4.57

$(3.54,5.91)$

$<0.001$

Reference

4.71 (3.87,

5.73)

$<0.001$

$1.11(0.86$,

1.41)

0.081

1.62 (1.04,

2.50)

.




\section{Cureus}

Dependent functional health status prior to surgery

$\begin{array}{lllll}\text { Yes } & 76(3.1) & 31(40.8) & 45(59.2) & \begin{array}{l}2.48(1.56, \\ 3.94)\end{array} \\ & & & & <0.001 \\ \text { No } & 2384 & 1503(63.0) & 881(37.0) & \text { Reference }\end{array}$

History of severe COPD

$\begin{array}{lllll}\text { Yes } & 471(19.1) & 251(53.3) & 220(46.7) & \begin{array}{l}1.58(1.29, \\ 1.93)\end{array} \\ & & & & <0.001 \\ \text { No } & 1997 & 1284(64.3) & 713(35.7) & \text { Reference }\end{array}$

Congestive heart failure in 30 days prior to surgery

$\begin{array}{lllcl}\text { Yes } & 56(2.3) & 18(32.1) & 38(67.9) & \begin{array}{l}3.58(2.03, \\ 6.31)\end{array} \\ & & & & <0.001 \\ \text { No } & 2412 & 1517(62.9) & 895(37.1) & \text { Reference }\end{array}$

Hypertension requiring medication

Yes

$\begin{array}{lllll}1959 & 1196(61.0) & 763(39.0) & 1.27(1.04, & \\ (79.4) & & & 1.56) & 0.022 \\ & & 170(33.4) & \text { Reference } & \end{array}$

Currently on dialysis (pre-op)

$\begin{array}{llccc}\text { Yes } & 43(1.7) & 22(51.2) & 21(48.8) & \begin{array}{l}1.58(0.87, \\ 2.90)\end{array} \\ & & & & 0.136 \\ \text { No } & 2425 & 1513(62.4) & 912(37.6) & \text { Reference }\end{array}$

Steroid use for chronic condition

\begin{tabular}{|c|c|c|c|c|c|}
\hline \multirow[t]{2}{*}{ Yes } & $94(3.8)$ & $54(57.4)$ & $40(42.6)$ & $\begin{array}{l}1.23 \\
(0.81,1.87)\end{array}$ & \multirow{3}{*}{0.334} \\
\hline & & & & & \\
\hline No & $\begin{array}{l}2374 \\
(96.2)\end{array}$ & $1481(62.4)$ & $893(37.6)$ & Reference & \\
\hline
\end{tabular}

Preoperative transfusion

$\begin{array}{lllll}\text { Yes } & 70(2.8) & 12(17.1) & 58(82.9) & \begin{array}{l}8.41(4.49, \\ 15.75)\end{array} \\ & & & & <0.001 \\ \text { No } & 2398 & 1523(63.5) & 875(36.5) & \text { Reference }\end{array}$

Systemic sepsis

Yes

$93(3.8) \quad 20(21.5)$

$73(78.5)$

$6.43(3.89$, 


\section{Cureus}

No

2375

(96.2)
10.62)

Wound classification

Clean

2447

$1529(62.5)$

$918(37.5)$

$0.24(0.09$, $0.62)$

0.003

Not clean

$21(0.9) \quad 6(28.6)$

$15(71.4)$

Reference

Intra-operative factors:

Prior abdominal aortic surgery

$$
\text { Yes }
$$

$659(29.7) \quad 396(60.1)$

$263(39.9)$

$1.17(0.97$ 1.41)

0.102

No

1562
$(70.3)$$\quad 996(63.8)$

$566(36.2)$

Reference

Surgeon's specialty

Vascular

2400

$1504(62.7)$

$896(37.3)$

Reference

Non-vascular

$68(2.8)$

$31(45.6)$

$37(54.4)$

$2.00(1.23$

3.25)

Principal operative procedure

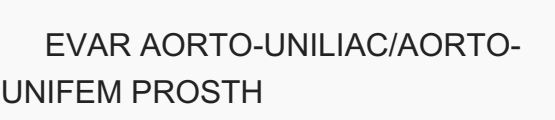

$105(4.3)$

$58(55.2)$

$47(44.8)$

1.35

(0.90,

2.02)

$2.19(1.52$,

3.14)

Reference

EVAR W/AORTO-AORTIC TUBE
EROSTH
EVAR W/MDLR BFRC PROSTH 1
LIMB

EVAR WIAORTO-AORTIC TUBE
PROSTH
EVAR W/MDLR BFRC PROSTH 1
LIMB

$134(5.4) \quad 58(43.3)$

$76(56.7)$

$409(37.5)$

1091
$(44.2)$

$682(62.5)$

$793(32.1)$

$549(69.2)$

$244(30.8)$

0.74 (0.61,

0.90)

1.45 (1.07,

1.96)

$1.32(0.93$

1.87) VSL

PLMT XTN PROSTH EVAR/DSJ $1^{\text {ST }}$

147 (6.0)

82 (55.8)

$65(44.2)$

Principal anesthesia technique

General

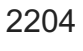

(89.4)

$1362(61.8)$

$842(38.2)$

$262(10.6) \quad 172(65.6)$

$90(34.4)$
$1.18(0.90$

1.55)

0.225
Non- general

ASA classification 


\section{Cureus}

$\begin{array}{lllll}\text { 1-2, no disturbance-mild disturbance } & 92(3.7) & 75(81.5) & 17(18.5) & \text { Reference } \\ & & & & \\ \text { 3, severe disturbance } & 1443 & 1008(69.9) & 435(30.1) & 1.90(1.11, \\ & (58.7) & & & 3.26) \\ \text { 4-5, life-threatening-moribund } & 924(37.6) & 448(48.5) & 476(51.5) & 4.69(2.73,\end{array}$

Rupture of aneurysm

$\begin{array}{lllll}\text { Yes } & 10(0.4) & 1(10.0) & 9(90.0) & \begin{array}{l}14.91 \\ (1.89, \\ 117.67)\end{array} \\ \text { No } & & & & \\ & & & & \\ \text { Reference }\end{array}$

Aneurysm diameter (cm)

$\leq 5.0$
$5.0-5.5$
$5.5-6.0$
$>6.0$

\begin{tabular}{|c|c|c|c|c|}
\hline $\begin{array}{c}576 \\
(24.6)\end{array}$ & 356 (61.8) & $220(38.2)$ & Reference & \\
\hline 667 (28.5) & $479(71.8)$ & $188(28.2)$ & $\begin{array}{l}0.64(0.50 \\
0.81)\end{array}$ & \\
\hline $455(19.4)$ & $309(67.9)$ & $146(32.1)$ & $\begin{array}{l}0.77(0.59 \\
0.99)\end{array}$ & \\
\hline $643(27.5)$ & 334 (51.9) & $309(48.1)$ & $\begin{array}{l}1.50(1.19 \\
1.88)\end{array}$ & \\
\hline
\end{tabular}

Proximal aneurysm extent

Infrarenal 2074

(89.6)

$1359(65.5)$

$241(10.4) \quad 99(41.1)$

Others (Juxta-, para-, supra-renal, type IV thoracoabdominal aneurysm)

Distal extent

Aortic

Common iliac

External iliac

Internal iliac

Access vessels (conduit, repair)

Yes

$129(6.7) \quad 69(53.5)$

$186(9.8) \quad 95(51.1)$
$715(34.5)$

$142(58.9)$

$258(28.6)$

$248(35.9)$

$60(46.5)$

$91(48.9)$

58 (56.9)

$2.25(1.51$ 3.35)

$<0.001$ 


\section{Cureus}

No

Access

Attempted percutaneous access

converted to open cutdown

Bilateral groin cutdown

One groin cutdown

Percutaneous bilateral

Main body device

Cook Zenith Fenestrated

Regular EVARs

Iliac branched device

Yes

No

Aortic (bare metal) stent

Yes

No

Renal stent

Yes

No

Hypogastric embolization

Yes

No

Hypogastric revascularization

Yes
(95.9)

$1491(63.0)$

$857(37)$

Reference

$1.40(0.59$,

$3.30)$

$1.48(1.25$,

1.76)

$1.29(0.96$,

1.73)

1169

(47.7)

$782(66.9)$

$387(33.1)$

Reference
$74(3.1) \quad 33(44.6) \quad 41(55.4)$

2350

(96.9)

$1486(63.2)$

$864(36.8)$

$158(44.3)$

$775(36.7)$

2111

(85.5)

1336 (63.3)

$88(52.4)$

$168(6.8) \quad 80(47.6)$

2300

(93.2)

1455 (63.3)

845(36.7)

$122(64.9)$

3.35 (2.45,

4.57)

$<0.001$

2280

(92.4)

1469 (64.4)

$811(35.6)$

Reference

1.89 (1.38, 2.59) $<0.001$

Reference

1.25 (0.92, 1.71)

2293

(92.9)

1435 (62.6)

$858(37.4)$

Reference

1.37 (1.09, 


\section{Cureus}

No

2316

(93.8)

$1480(63.9)$

$836(36.1)$

Lower extremity revascularization

Yes

No

$113(4.6) \quad 52(46.0)$

2355

(95.4)

$1483(63.0)$

$61(54.0)$

$872(37.0)$

$2.00(1.37$

2.92) $<0.001$

Ischemic colitis

$$
\text { Yes }
$$

No

Lower extremity ischemia

Yes

No

Total operation time (minutes)

$<90$

$90-120$

$120-180$

$\geq 180$

Postoperative factors:

Discharge destination

\section{Expired}

Home

Other

Open wound/wound infection
$41(1.7)$

2427

(98.3)

651 (26.4) 471 (72.3)

629 (25.5) $431(68.5)$

$713(28.8) \quad 442(62.0)$

475 (19.3) 191 (40.2)
$23(56.1)$

$910(37.5)$

$180(27.7)$

$198(31.5)$

$271(38.0)$

$284(59.8)$
42.11

(5.71,

310.39)

$<0.001$

Reference

$2.13(1.14$ 3.97) 0.017

Reference

Reference

$1.20(0.95$, 1.53)

$1.60(1.28,<0.001$ 2.02)

3.89 (3.03, $5.00)$
8.33 (4.42, 15.72)

Reference $\quad<0.001$

$\begin{array}{llll}\begin{array}{l}2173 \\ (88.1)\end{array} & 1439(66.2) & 734(33.8) & \text { Reference } \\ 228(9.3) & 83(36.4) & 145(63.6) & \begin{array}{l}3.43(2.58, \\ 4.55)\end{array} \\ & & & \\ & & & \\ 31(1.3) & 14(45.2) & 17(54.8) & 2.02(0.99, \\ & & & 4.11)\end{array}$




\section{Cureus}

No

2437

(98.7)

$1521(62.4)$

$916(37.6)$

Reference

0.054

Superficial surgical site occurrence

Superficial surgical incisional SS

2442

No Complication

(98.9)

$1521(62.3)$

$12(46.2)$

$1.42(0.65$ 3.07)

$921(37.7)$

Reference

Deep incisional SSI

Deep incisional SSI

$8(0.3)$

$1(12.5)$

$7(87.5)$

11.59

(1.42,

94.32)

0.022

No complication

2460

(99.7)

$1534(62.4)$

$926(37.6)$

Reference

Wound disruption

Wound disruption

$3(0.1)$

$1(33.3)$

$2(66.7)$

$3.30(0.30$, 36.38)

No complication

2465

(99.9)

$1534(62.2)$

$931(37.8)$

Reference

Pneumonia

Pneumonia

$44(1.8)$

$4(9.1)$

$40(90.9)$

17.14

(6.11,

48.08)

$<0.001$

No complication

2424

(98.2)

1531 (63.2)

$893(36.8)$

Reference

Pulmonary embolism

Pulmonary embolism

$8(0.3)$

$1(12.5)$

$7(87.5)$

2460

No complication

(99.7)

$1534(62.4)$

$926(37.6)$

11.59

(1.42,

94.32)

0.022

Reference

Urinary tract infection

Urinary tract infection

$24(1.0)$

$11(45.8)$

$13(54.2)$

$1.96(0.87$, 4.39)

0.103-

No complication

2444

$1524(62.4)$

$920(37.6)$

Reference

(99.0)

Acute renal failure

Acute renal failure

$24(1.0) \quad 1(4.2)$

$23(95.8)$

(5.23, 


\section{Cureus}

No complication

CVA/stroke with neurological deficit

CVA/stroke

No complication

Cardiac arrest requiring CPR

Cardiac arrest requiring CPR

No complication

Myocardial infarction

Myocardial infarction

No complication

Bleeding transfusion

Transfusion

intraoperative/postoperative

No complication

DVT/thrombophlebitis

DVT requiring therapy

No complication

Sepsis

Sepsis

No complication

Return to operation room

Yes

No

\section{4}

(99.0)

$1534(62.8)$

$11(0.5) \quad 2(18.2)$

$9(81.8)$

2457

(99.5)

$1533(62.4)$

$924(37.6)$

7.47 (1.61, $34.63)$ 0.010

Reference

$4.29(2.12$ 8.65) $<0.001$

2429(98.4) 1524 (62.7)

$905(37.3)$

Reference

$9.64(4.03$, 23.04) $<0.001$

2428

(98.4)

1529 (63.0)

899 (37.0)

Reference

9.16 (6.80, 12.33) $<0.001$

$\begin{array}{llll}2159 & 1476(68.4) & 683(31.6) & \text { Reference }\end{array}$

9.99 (2.23, 44.71) 0.003

$2454 \quad 1533(62.5) \quad 921(37.5) \quad$ Reference

$\begin{array}{lll}14(0.6) & 4(28.6) & 10(71.4) \\ 2454 & 1531(62.4) & 923(37.6) \\ (99.4) & \end{array}$

4.15 (1.30, 13.26) 0.017

Reference

$\begin{array}{ll}128(5.2) & 46(35.9) \\ 2340 & 1489(63.6) \\ (94.8) & \end{array}$

$82(64.1)$

851 (36.4)
$3.12(2.15$ 4.52) $<0.001$

Reference 


\section{Cureus}

Unplanned readmission

$\begin{array}{lllcr}\text { Yes } & 188(7.6) & 82(43.6) & 106(56.4) & \begin{array}{l}2.27(1.68, \\ 3.07)\end{array} \\ & & & & <0.001 \\ \text { No } & 2280 & 1453(63.7) & 827(36.3) & \text { Reference }\end{array}$

* All odds ratios and p-values are from binomial logistic regression modeling ICU LOS $\geq 1$, exact logistic regression used as needed. When an association is significant ( $p$-value), odds ratios with 95\% confidence limits not including 1 are considered significant.

\section{TABLE 1: Patient characteristics by ICU LOS}

ICU LOS, intensive care unit length of stay; OR, odds ratio; $\mathrm{Cl}$, confidence interval; BMI, body mass index; COPD, chronic obstructive pulmonary disease; EVAR, endovascular aneurysm repair; EVAR AORTO-UNILIAC/AORTO-UNIFEM PROSTH, EVAR with aortouniiliac/aorto-unifemoral prosthesis; EVAR WIAORTO-AORTIC TUBE PROSTH, EVAR with aorto-aortic tube prosthesis; EVAR W/MDLR BFRC PROSTHE 1 LIMB, EVAR with modular bifurcated prosthesis with one docking limb; EVAR W/MDLR BFRC PROSTH 2 LIMBS, EVAR with modular bifurcated prosthesis with two docking limbs; EVAR W/UNIBDY BFRC PROSTH, EVAR with unibody bifurcated prosthesis; PLMT XTN PROSTH EVAR/DSJ 1st VSL, placement of extension prosthesis for endovascular repair of infrarenal abdominal aortic or iliac aneurysm, initial vessel; ASA, American Society of Anesthesiologists; SSI, surgical site infection; CVA, cerebrovascular accident; CPR, cardiopulmonary resuscitation; DVT, deep vein thrombosis

\section{Multivariable analysis}

The following factors were found to have significant association with ICU LOS $\geqslant 1$ day: ruptured AAA (OR 3.88, CI: $1.97-7.65, p<0.001)$, aneurysm $\leqslant 5 \mathrm{~cm}$ (vs. $>6.0 \mathrm{~cm}$ : OR 1.50, CI: $1.12-2.02, p<$ 0.001 ), ASA score of 4-5 (vs. $1-2$ : OR 2.82 , CI: $1.50-5.31, p<0.001$ ), operative time $\geqslant 180$ minutes (vs <90 minutes: OR 2.10, CI: 1.51-2.93, $p<0.001$ ), bilateral groin cut downs (vs. percutaneous bilateral: OR 1.37, CI: 1.10-1.71, $p=0.006$ ), non-infrarenal AAA (OR 1.65, CI: 1.16-2.35, $p=$ 0.005 ), renal artery stent (OR 2.13, CI: 1.42-3.21, $p<0.001$ ), aortic stent (OR 2.39, CI: 1.60-3.55, $p<0.001$ ), emergency surgery (OR 2.56, CI: 1.94-3.38, $\mathrm{p}<0.001$ ), need for blood transfusion (OR 3.11, CI: 2.08-4.65, $p<0.001$ ), postoperative pneumonia (OR 7.04, 1.95-25.45, $p=0.003$ ), and unplanned readmission (OR 1.98, CI: 1.35-2.91, $p<0.001$; Table 2).

\section{Risk factor}

OR $(95 \% \mathrm{Cl})^{*}$

P-value*

Elective surgery

Yes

Reference

No

$2.56(1.94,3.38)$

Indication for Surgery

Ruptured AAA

$3.88(1.97,7.65)$

Non-ruptured AAA

Reference

Operation time (minutes)

$<90$

Reference

$90-120$ 


\section{Cureus}

$120-180$

$\geq 180$

Aneurysm diameter $(\mathrm{cm})$

$\leq 5.0$

$5.0-5.5$

$5.5-6.0$

$>6.0$

ASA classification

1-2, no disturbance-mild disturbance

3 , severe disturbance

4-5, life-threatening-moribund

Access

Attempted percutaneous access converted to open cut-down

Bilateral groin cut-down

One groin cut-down

Percutaneous bilateral

Proximal aneurysm extent

Infrarenal

Others (Juxta-, para-, supra-renal, type IV thoracoabdominal aneurysm)

Renal stent

Yes

No

Aortic (bare metal) stent

Yes

No

Pneumonia

Pneumonia

No complication

Bleeding transfusion

Transfusions/ intraop/postop

No complication
$1.27(0.95,1.70)$

$2.10(1.51,2.93)$

$1.50(1.12,2.02)$

$0.84(0.62,1.12)$

$0.86(0.63,1.19)$

$<0.001$

Reference

Reference

$1.69(0.90,3.14) \quad<0.001$

$2.82(1.50,5.31)$

$0.44(0.10,1.90)$

$1.37(1.10,1.71)$

$0.84(0.57,1.24)$

Reference

\section{Reference}

$1.65(1.12,2.35)$

0.005

$2.13(1.42,3.21)$

Reference

$2.39(1.60,3.55)$

Reference

$<0.001$

$7.04(1.95,25.45)$

Reference

$3.11(2.08,4.65)$

Reference
$<0.001$ 


\section{Cureus}

Unplanned readmission

Yes

* Multivariable logistic regression model for ICU LOS $\geq 1$; odds ratios and p-values are adjusted for all other variables in the model/table.

\section{TABLE 2: Final multivariable model for ICU LOS $\geq 1$ day}

ICU LOS, intensive care unit length of stay; OR, odds ratio; Cl, confidence interval; AAA, abdominal aortic aneurysm; ASA, American Society of Anesthesiologists

\section{Predicted probability}

The probability of ICU LOS $\geqslant 1$ day was calculated for each factor identified from multivariable analysis. The probability of ICU LOS $\geqslant 1$ day was $11.73 \%$ for patients who underwent bilateral groin cut down, $12.72 \%$ for patients with aneurysm diameter $\leqslant 5 \mathrm{~cm}, 13.82 \%$ for patients who had non-infrarenal proximal aneurysm, $16.14 \%$ for patients who needed unplanned readmission, $16.95 \%$ for patients whose operative time was $\geqslant 180$ minutes, $17.14 \%$ for patients who needed renal stent, $18.81 \%$ for patients who needed aortic stent, $19.90 \%$ for patients who underwent EVAR as non-elective surgery, 21.48\% for patients who had an ASA classification of 4-5, 23.17\% for patients who needed transfusion (intra/postoperative), $27.36 \%$ for patients who had ruptured aneurysm as indication for surgery, and $40.57 \%$ for patients who had postoperative pneumonia. Patients who had all of these factors have a $99.99 \%$ probability of having ICU LOS $\geqslant 1$ day after EVAR. Patients without any of these factors had a probability of $8.84 \%$ at baseline (Table 3). 


\section{Cureus}



\section{TABLE 3: Predicted probabilities of ICU LOS $\geq 1$ from a multivariable model}

\section{Discussion}

This analysis of a large, multi-institutional database of all EVARs performed across the US aims to determine the factors associated with increased length of ICU stay during the postoperative period. It identifies the following risk factors: ruptured AAA, emergent surgery, longer operative time, higher ASA score, bilateral groin cut-downs, extension of aneurysm into the supra-renal aortic segment, need for aortic or renal stents, postoperative pneumonia, bleeding and unplanned readmission to hospital. The predicted probability model shows that in the absence of these risk factors, the probability of prolonged ICU admission is $8.8 \%$, and in the presence of all of these risk factors, the predicted probability of prolonged ICU stay is $99.99 \%$. Presence of post-operative pneumonia itself is associated with a $41 \%$ probability of prolonged ICU admission. These findings have potentially important implications for the management of EVAR patients.

Our study shows several important factors that contribute to prolonged ICU stay after EVAR (Table 2). While some of these factors can be considered modifiable, several of them cannot be modified. For example, variables such as ruptured AAA, high ASA scores and juxta-renal AAAs 
cannot be changed. On the other hand, factors such as bilateral femoral cut-downs, prolonged operative time and postoperative pneumonia can be considered potentially preventable. For factors that cannot be modified, this study highlights the importance of recognizing patients who are at high risk for requiring prolonged ICU stay. It also highlights risk factors that can be prevented to reduce ICU LOS. Postoperative pneumonia is associated with an extremely high risk for prolonged ICU stay after EVAR.

Endovascular aneurysm repair vs open repair (EVAR trial 1) and the Dutch randomized trial comparing conventional and endovascular repair of AAA (DREAM Trial) have established EVAR as a safe and effective alternative surgical treatment for AAA [2-3]. Among many advantages of EVAR over open repair are the reduced operative time, length of ICU stay, length of hospital stay, and early ambulation. In addition, EVAR is more cost-effective as compared to open AAA repair. While the cost of endografts is the major contributor toward the overall cost of EVAR, major reduction in the cost comes from reduced ICU LOS and hospital LOS. During the past decade, the overall cost associated with EVAR has improved, and most of this cost reduction comes from reduced LOS [12]. Our intent for this study was to examine all factors associated with ICU LOS and to focus on those factors, which are deemed preventable. Our study shows that the development of postoperative pneumonia is a significant preventable factor. Strategies to improve preventable factors may help us reduce the ICU LOS, associated morbidities, and overall cost of EVAR. Prolonged ICU LOS after EVAR itself has been associated with the development of serious complications, including death [5]. With an increasing focus on reducing postoperative complications and stress on using cost-effective strategies, our analysis identifies an area of improvement for patients undergoing EVAR. Postoperative complications impose a tremendous financial burden on both the patient and the healthcare system [13-14]. A retrospective review of the veteran affairs (VA) surgical patients showed that the estimated excess cost from postoperative complications ranged from $\$ 8,338$ for superficial surgical site infection to $\$ 29,595$ for failure to wean from ventilation within 48 hours. For pneumonia itself, the estimated excess cost was $\$ 12,798$ [14]. Postoperative complications are highly associated with readmission, and readmission comes with additional cost. Lawson et al. show that by $5 \%$ reduction in postoperative complications, a potential 2,092 readmissions can be prevented, saving Medicare 31 million dollars per year [15].

Patients undergoing major vascular surgery operations are at significantly high risk for developing postoperative complications compared to those who undergo other major surgical operations [16]. Postoperative morbidity is associated with a significantly high risk of mortality [17]. Association of postoperative morbidity is, in fact, higher than that of baseline comorbid disease [18]. While the other complications after EVAR have been described in literature before, there is sparse literature on the impact of postoperative pneumonia on the overall prognosis. A retrospective review of all vascular surgery operations performed across the US has shown that postoperative pneumonia is associated with a significantly high risk of developing end-organ dysfunction, 30-day mortality, prolonged length of hospital stay and hospital readmission [19]. Our study shows that the development of postoperative pneumonia is associated with increased LOS in the ICU in the postoperative period. These findings are important, as postoperative pneumonia can be considered preventable for elective operations. Katsura et al. have shown that respiratory muscle training in the preoperative time period can reduce the incidence of postoperative pulmonary complications among patients undergoing cardiac and abdominal operations [20]. Guay et al. have demonstrated that intraoperative use of low volume ventilation reduces the incidence of postoperative respiratory complications

[21]. Unfortunately, ACS-NSQIP data does not have information available regarding the ventilator settings during the operations. Popping et al. document the benefit of epidural analgesia on decreasing postoperative pulmonary morbidity [22]. Ireland et al. have stressed the importance of continuous airway pressure during the postoperative period to prevent postoperative morbidity [23]. Such strategies can be employed to prevent postoperative pneumonia and hence the complications associated with it. There is evidence to support that 
development of pneumonia prevention bundles by hospitals reduces the incidence of pneumonia [24-25]. The findings from our study, in combination with the above-mentioned literature, make the case for employing all pneumonia prevention strategies for EVAR patients to reduce the incidence of this complication and the associated increased length of ICU stay. As shown by Greenblatt et al., reducing the incidence of pneumonia after aortic surgery can also help reduce the incidence of unplanned readmission to the hospital [26].

This study has several limitations. It is a retrospective analysis where findings are limited to those hospitals that were enrolled in ACS-NSQIP in 2015. In addition, the data is not allinclusive, and it is a self-reporting registry. We lack information about the documented reason for ICU admission. The strengths of this study are that it is based on the largest surgical database in the US. The findings from this database in previously published literature have been shown to be reliable and reproducible in subsequent studies. The large volume of patients in the dataset provides a powerful tool for meaningful statistical analysis. It includes large, tertiary care academic medical centers and small community hospitals, hence providing data from a broad variety of sources. Correlation of pneumonia with increased ICU LOS and other morbidities correlates with previously published literature. Our data shows that the occurrence of more than one complication is associated with longer length of ICU stay.

\section{Conclusions}

With EVAR becoming the predominant modality for surgical treatment of AAA, attention should be paid to factors associated with increased morbidity and mortality after EVAR. Prolonged ICU LOS is associated with poor outcomes and increased cost. This study identifies the factors associated with prolonged ICU LOS after EVAR and identifies that postoperative pneumonia is associated with a significant risk of increased ICU LOS.

\section{Additional Information}

\section{Disclosures}

Human subjects: All authors have confirmed that this study did not involve human participants or tissue. Animal subjects: All authors have confirmed that this study did not involve animal subjects or tissue. Conflicts of interest: In compliance with the ICMJE uniform disclosure form, all authors declare the following: Payment/services info: All authors have declared that no financial support was received from any organization for the submitted work.

Financial relationships: All authors have declared that they have no financial relationships at present or within the previous three years with any organizations that might have an interest in the submitted work. Other relationships: All authors have declared that there are no other relationships or activities that could appear to have influenced the submitted work.

\section{References}

1. Parodi JC, Palmaz JC, Barone HD: Transfemoral intraluminal graft implantation for abdominal aortic aneurysms. Ann Vasc Surg. 1991, 5:491-9. 10.1007/BF02015271

2. Greenhalgh RM, Brown LC, Kwong GP, Powell JT, Thompson SG; EVAR trial participants: Comparison of endovascular aneurysm repair with open repair in patients with abdominal aortic aneurysm (EVAR trial 1), 30-day operative mortality results: randomised controlled trial. Lancet. 2004, 364:843-8. 10.1016/S0140-6736(04)16979-1

3. Prinssen M, Verhoeven EL, Buth J, et al.: A randomized trial comparing conventional and endovascular repair of abdominal aortic aneurysms. N Engl J Med. 2004, 351:1607-18. 10.1056/NEJMoa042002

4. Lachat ML, Pecoraro F, Mayer D, et al.: Outpatient endovascular aortic aneurysm repair: experience in 100 consecutive patients. Ann Surg. 2013, 258:754-8.

10.1097/SLA.0b013e3182a617f1

5. Cadili A, Turnbull R, Hervas-Malo M, Ghosh S, Chyczij H: Identifying patients with AAA with 
the highest risk following endovascular repair. Vasc Endovascular Surg. 2012, 46:455-9.

$10.1177 / 1538574412450752$

6. American College of Surgeons National Surgical Quality Improvement Program. User guide for the 2015 ACS NSQIP participant use data file (PUF). (2017). Accessed: April 16, 2019: https://www.facs.org/ /media/files/quality\%20programs/nsqip/nsqip_puf_user_guide_2015.ashx.

7. Shiloach M, Frencher SK Jr., Steeger JE, et al.: Toward robust information: data quality and inter-rater reliability in the American College of Surgeons National Surgical Quality Improvement Program. J Am Coll Surg. 2010, 210:6-16. 10.1016/j.jamcollsurg.2009.09.031

8. Daley J, Khuri SF, Henderson W, et al.: Risk adjustment of the postoperative morbidity rate for the comparative assessment of the quality of surgical care: results of the National Veterans Affairs surgical risk study. J Am Coll Surg. 1997, 185:328-40. 10.1016/S1072-7515(01)00939-5

9. Bilimoria K, Cohen M, Ingraham A, Bentrem D, Richard K, Hall B, Ko C: Effect of postdischarge morbidity and mortality on comparisons of hospital surgical quality. Ann Surg. 2010, 252:183-

90. 10.1097/SLA.0b013e3181e4846e

10. Khuri SF, Daley J, Henderson W, et al.: Risk adjustment of the postoperative mortality rate for the comparative assessment of the quality of surgical care: results of the National Veterans Affairs Surgical Risk Study. J Am Coll Surg. 1997, 185:315-27. 10.1016/S1072-7515(01)00938-3

11. Khuri SF, Daley J, Henderson W, et al.: The National Veterans Administration Surgical Risk Study: risk adjustment for the comparative assessment of the quality of surgical care. J Am Coll Surg. 1995, 180:519-31.

12. Salzler GG, Meltzer AJ, Mao J, Isaacs A, Connolly PH, Schneider DB, Sedrakyan A: Characterizing the evolution of perioperative outcomes and costs of endovascular abdominal aortic aneurysm repair. J Vasc Surg. 2015, 62:1134-9. 10.1016/j.jvs.2015.06.138

13. Dimick JB, Chen SL, Taheri PA, Henderson WG, Khuri SF, Campbell DA Jr: Hospital costs associated with surgical complications: a report from the private-sector National Surgical Quality Improvement Program. J Am Coll Surg. 2004, 199:531-7.

10.1016/j.jamcollsurg.2004.05.276

14. Carey K, Stefos T, Shibei Z, Borzecki AM, Rosen AK: Excess costs attributable to postoperative complications. Med Care Res Rev. 2011, 68:490-503. 10.1177/1077558710396378

15. Lawson EH, Hall BL, Louie R, et al.: Association between occurrence of a postoperative complication and readmission: implications for quality improvement and cost savings. Ann Surg. 2013, 258:10-8. 10.1097/SLA.0b013e31828e3ac3

16. Gawande AA, Thomas EJ, Zinner MJ, Brennan TA: The incidence and nature of surgical adverse events in Colorado and Utah in 1992. Surgery. 1999, 216:66-75.

10.1067/msy.1999.98664

17. Khuri SF, Henderson WG, DePalma RG, Mosca C, Healey NA, Kumbhani DJ: Determinants of long-term survival after major surgery and the adverse effect of postoperative complications. Ann Surg. 2005, 242:326-41. 10.1097/01.sla.0000179621.33268.83

18. Silber JH, Rosenbaum PR, Trudeau ME, et al.: Changes in prognosis after the first postoperative complication. Med Care. 2005, 43:122-31.

19. Bennett KM, Kent KC, Schumacher J, Greenberg CC, Scarborough JE: Targeting the most important complications in vascular surgery. J Vasc Surg. 2017, 65:793-803.

10.1016/j.jvs.2016.08.107

20. Katsura M, Kuriyama A, Takeshima T, Fukuhara S, Furukawa TA: Preoperative inspiratory muscle training for postoperative pulmonary complications in adults undergoing cardiac and major abdominal surgery. Cochrane Database Syst Rev. 2015, 10:CD010356.

10.1002/14651858.CD010356.pub2

21. Guay J, Ochroch EA: Intraoperative use of low volume ventilation to decrease postoperative mortality, mechanical ventilation, lengths of stay and lung injury in patients without acute lung injury. Cochrane Database Syst Rev. 2015, 12:CD011151.

10.1002/14651858.CD011151.pub2

22. Popping DM, Elia N, Van Aken HK, et al.: Impact of epidural analgesia on mortality and morbidity after surgery: systematic review and meta-analysis of randomized controlled trials. Ann Surg. 2014, 259:1056-67. 10.1097/SLA.0000000000000237

23. Ireland CJ, Chapman TM, Mathew SF, Herbison GP, Zacharias M: Continuous positive airway pressure (CPAP) during the postoperative period for prevention of postoperative morbidity and mortality following major abdominal surgery. Cochrane Database Syst Rev. 2014, 8:CD008930. 10.1002/14651858.CD008930.pub2 


\section{Cureus}

24. Cassidy MR, Rosenkranz P, McCabe K, Rosen JE, McAneny D: I COUGH: reducing postoperative pulmonary complications with a multidisciplinary patient care program. JAMA Surg. 2013, 148:740-5. 10.1001/jamasurg.2013.358

25. Wren SM, Martin M, Yoon JK, Bech F: Postoperative pneumonia-prevention program for the inpatient surgical ward. J Am Coll Surg. 2010, 210:491-5. 10.1016/j.jamcollsurg.2010.01.009

26. Greenblatt DY, Greenberg CC, Kind AJ, et al.: Causes and implications of readmission after abdominal aortic aneurysm repair. Ann Surg. 2012, 256:595-605.

10.1097/SLA.0b013e31826b4bfe 